\title{
ST-Segment Elevation Myocardial Infarction as a Result of Coronary Artery Ectasia-Related Intracoronary Thrombus in a Patient with Liver Cirrhosis
}

\author{
Ji Woong Roh, M.D., Eun Hyea Park, M.D., Joon Cheol Song, M.D., Young Seung Oh, M.D., Tong Yoon Kim, M.D., \\ Hyo Suk Kim, M.D., and Sungmin Lim, M.D.
}

Department of Internal Medicine, Bucheon St. Mary's Hospital, College of Medicine, The Catholic University of Korea, Bucheon, Korea

\begin{abstract}
Coronary artery ectasia (CAE) is a rare condition defined as the dilatation of coronary artery to at least 1.5 times larger than the normal adjacent coronary artery. Clinical manifestations of CAE vary, ranging from asymptomatic to ST-segment elevation myocardial infarction (STEMI). Because of its rarity and clinical diversity, the best treatment strategy and prognosis for CAE remain unclear. We describe a case of STEMI caused by intracoronary thrombus formation within an ectatic area in a patient with liver cirrhosis (LC). The patient was successfully managed by thrombus aspiration only, without balloon angioplasty or stent implantation, and maintained by dual antiplatelet therapy with aspirin and ticagrelor, a potent new P2Y12 inhibitor.
\end{abstract}

Key Words: antiplatelet agents; coronary artery ectasia; liver cirrhosis; ST-segment elevation myocardial infarction.

Coronary artery ectasia (CAE) is a rare disease defined as the dilation of the coronary artery to a size $\geq 1.5$ times greater than the diameter of the adjacent healthy reference segment.[1-3] The clinical features associated with CAE range from asymptomatic or stable angina to ST-segment elevation myocardial infarction (STEMI) or sudden cardiac death.[1] Although plaque rupture is a crucial mechanism of acute coronary syndrome (ACS) in patients with atherosclerotic coronary artery disease (CAD), the turbulent flow within the ectatic segment can induce thrombus formation, and distal embolization can also cause ACS.[4] Meanwhile, it is believed that liver cirrhosis has a protective role against atherosclerotic CAD because of some common characteristics in cirrhotic patients, such as coagulation abnormalities, decreased synthetic low-density lipoprotein (LDL) cholesterol, decreased vascular resistance, and low blood pressure.[5] Previous studies have described a lower prevalence of STEMI in patients with LC compared with that in the general populations.[6] In the present report, we describe a case of STEMI caused by ectasia-related intracoronary thrombus formation in an LC patient with thrombocytopenia and a prolonged prothrombin time $(\mathrm{PT})$.

\section{Case Report}

Received on October 7, 2015 Revised on November 4, 2015

Accepted on November 4, 2015

Correspondence to: Sungmin Lim, Department of Internal Medicine, Bucheon

St. Mary's Hospital, College of Medicine, The Catholic University of Korea, 327

Sosa-ro, Wonmi-gu, Bucheon 14647, Korea

Tel: +82-32-340-7641, Fax: +82-32-340-7227

E-mail: sungmin@catholic.ac.kr

*No potential conflict of interest relevant to this article was reported.
A 52-year-old man presented to our hospital emergency department complaining of sudden-onset ongoing retrosternal chest pain with diaphoresis for the previous 20 minutes. He had been diagnosed with primary hypertension 12 years previously and was taking amlodipine $5 \mathrm{mg}$ qd. He had also 


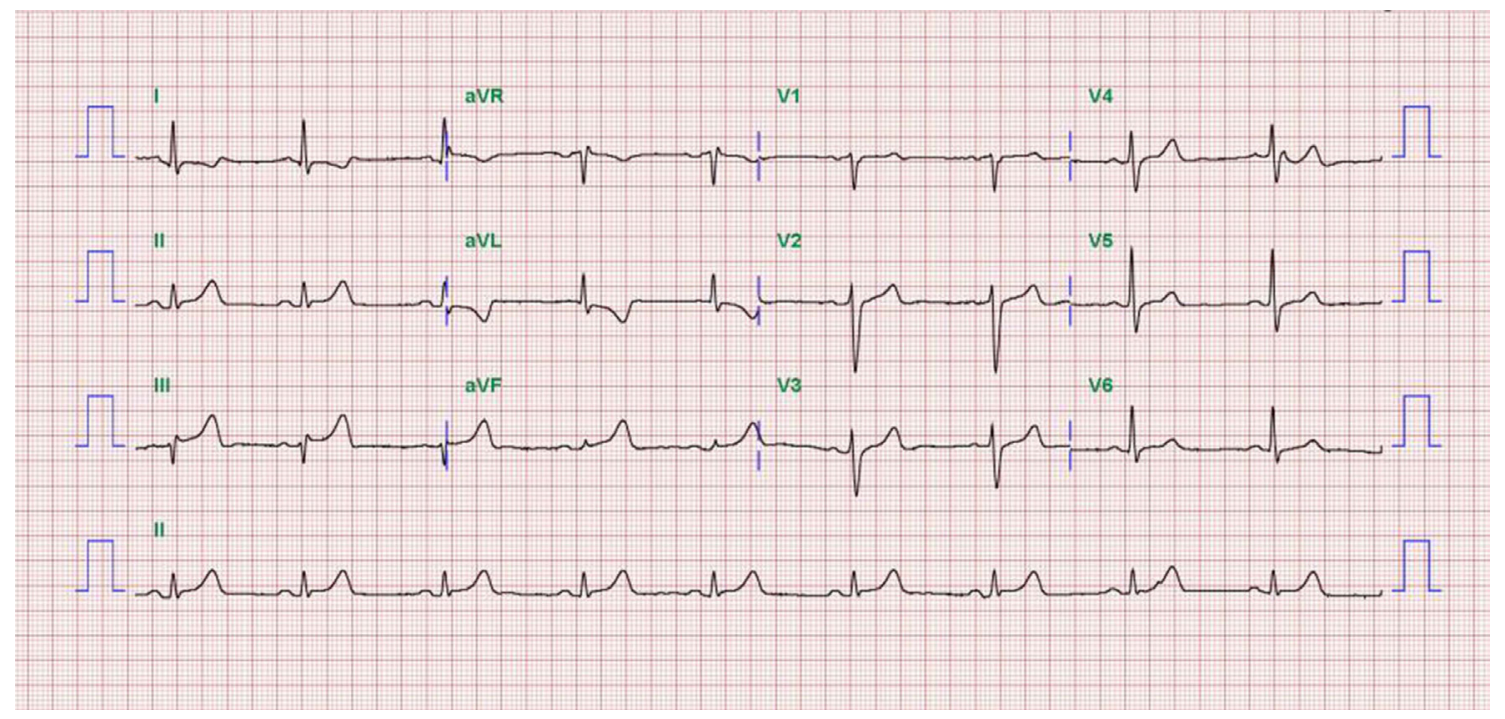

Fig. 1. Electrocardiograms at admission shows ST-segment elevation on the II, III, and aVF leads with reciprocal change on the I and aVL leads.
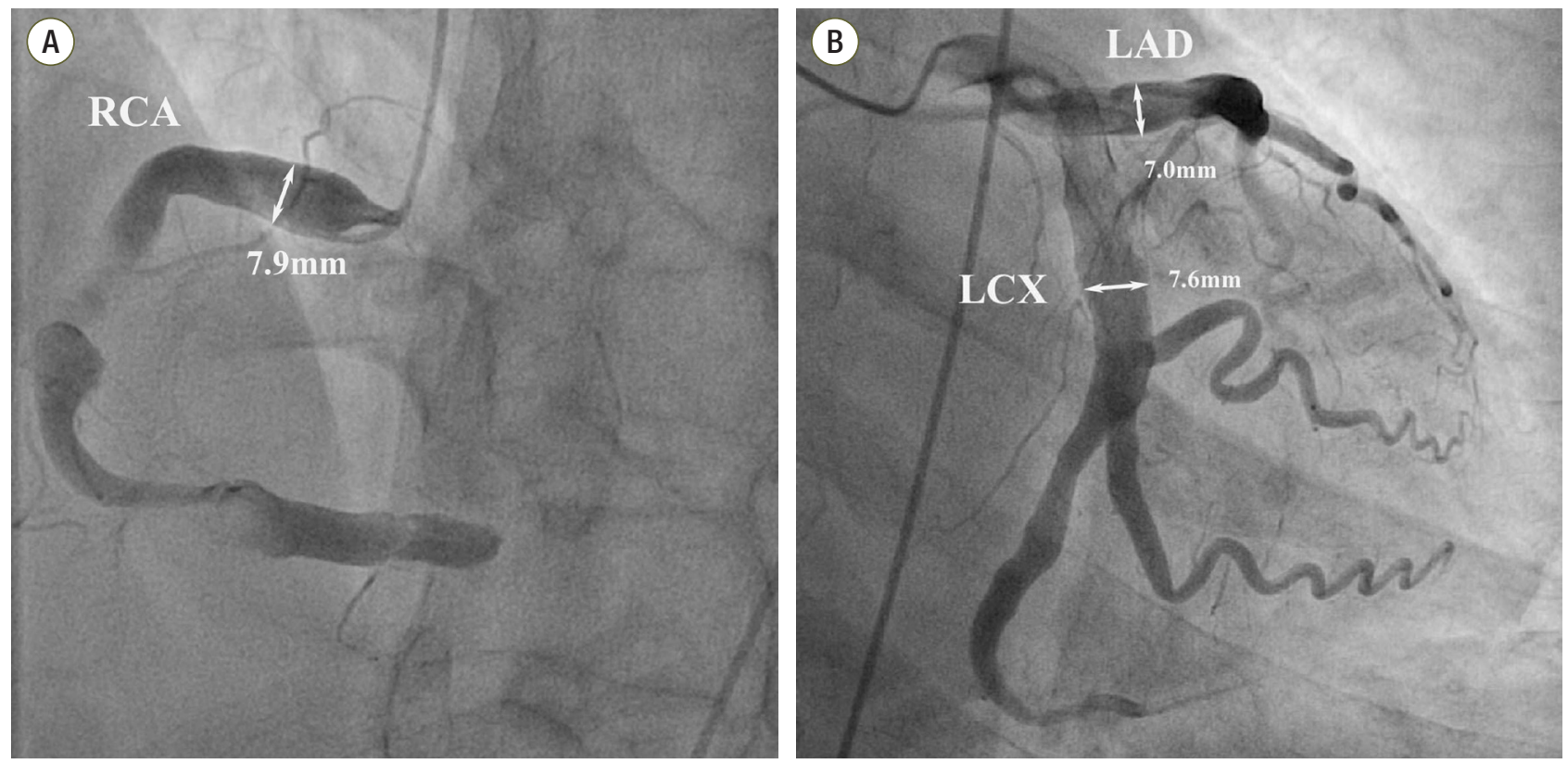

Fig. 2. Coronary angiography shows severe ectatic dilatation in all 3 mojor epicardial coronary arteries. (A) Distal right coronary artery was totally occluded by thrombus. Proximal right coronary artery segment diameter is $7.9 \mathrm{~mm}$, (B) proximal left anterior descending coronary artery segment diameter is $7.0 \mathrm{~mm}$ and the proximal left circumflex coronary artery is $7.6 \mathrm{~mm}$. RCA: right coronary artery; LAD: left anterior descending coronary artery; LCX: left circumflex coronary artery.

been diagnosed with LC caused by hepatitis B 5 years previously and was taking adefovir $10 \mathrm{mg}$ qd and lamivudine $100 \mathrm{mg}$ qd. He had never smoked and had no family history of CAD. On physical examination, his blood pressure was 90/50 $\mathrm{mmHg}$, and his heart rate was 54 beats/min. The patient's height was $178 \mathrm{~cm}$, and his body weight was $80 \mathrm{~kg}$.
His mental status was alert. His heart and lung sounds were nonspecific. Chest radiography was unremarkable, with no evidence of pulmonary edema. Electrocardiogram showed ST-segment elevation on the II, III, and aVF leads with reciprocal changes on the I and aVL leads (Fig. 1). Transthoracic echocardiogram revealed hypokinesia on the inferior 
wall and a left ventricular ejection fraction of 53\%. Laboratory findings showed a white blood cell count of $3,600 / \mathrm{mm}^{3}$, hemoglobin of $13.5 \mathrm{~g} / \mathrm{dL}$, platelet count of $41,000 / \mathrm{mm}^{3}$, creatinine of $1.1 \mathrm{mg} / \mathrm{dL}$, amino transferase/alanine aminotransferase of $24 / 21 \mathrm{U} / \mathrm{L}$, total bilirubin of $1.6 \mathrm{mg} / \mathrm{dL}$, albumin of $3.4 \mathrm{~g} / \mathrm{dL}$, and PT-international normalized ratio (INR) of 1.8 , indicating Child-Turcotte-Pugh class A. Cardiac biomarkers revealed creatine kinase $(\mathrm{CK})$ of $89 \mathrm{U} / \mathrm{L}, \mathrm{CK}-\mathrm{MB}$ of 1.76 $\mathrm{ng} / \mathrm{mL}$, and troponin-I of $<10 \mathrm{pg} / \mathrm{mL}$. The follow up levels showed CK-MB of $54.52 \mathrm{ng} / \mathrm{mL}$ and troponin-I of 16,014.0 $\mathrm{pg} / \mathrm{mL}$. The patient's lipid profile showed a total cholesterol level of $101 \mathrm{mg} / \mathrm{dL}$, triglycerides of $73 \mathrm{mg} / \mathrm{dL}$, high-density

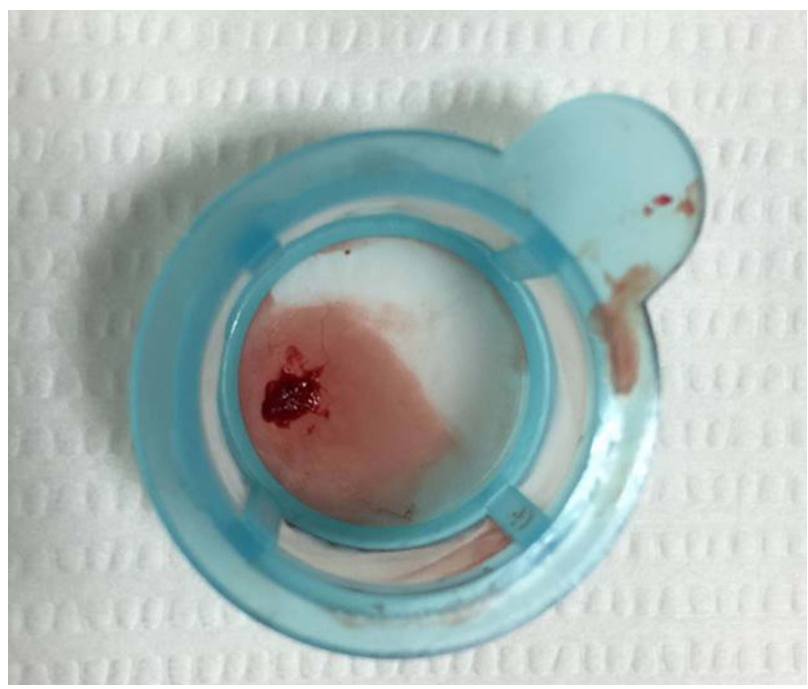

Fig. 3. There are aspirated red thrombi by thrombosuction device. lipoprotein cholesterol of $32 \mathrm{mg} / \mathrm{dL}$, and LDL cholesterol of $58 \mathrm{mg} / \mathrm{dL}$.

We diagnosed the patient with STEMI and decided to perform primary percutaneous coronary intervention. Although the bleeding risk of the patient appeared to be higher, we aimed at appropriate reperfusion preferentially and decided to manage the balance between antithrombotic efficacy and bleeding risk after the patient stabilized. Aspirin (300 mg loading dose) and ticagrelor (180 mg loading dose) were

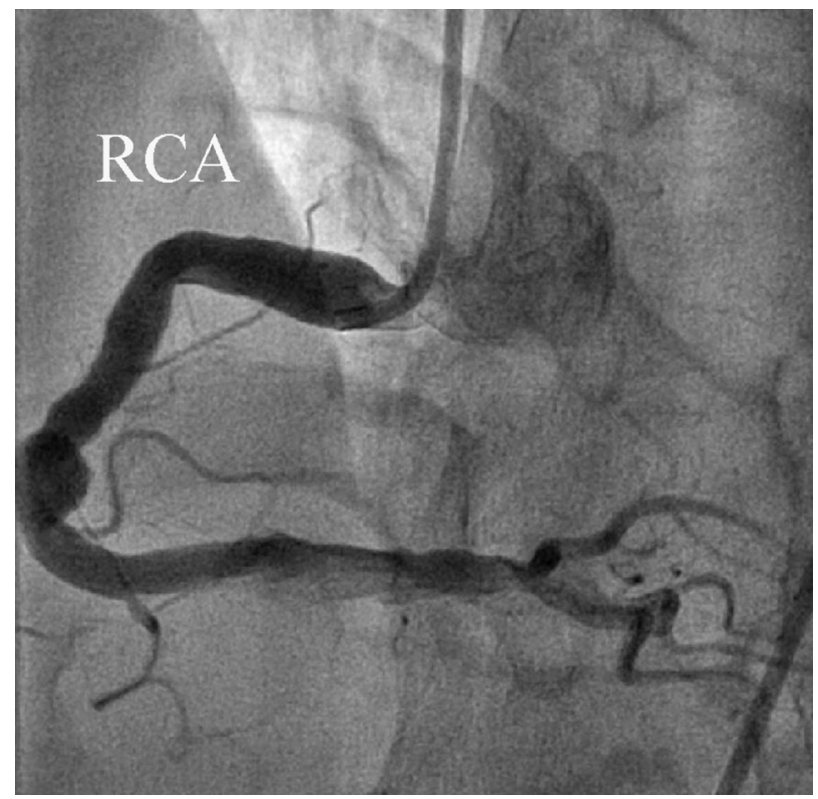

Fig. 4. After thrombosuction, right coronary artery flow was totally recovered.

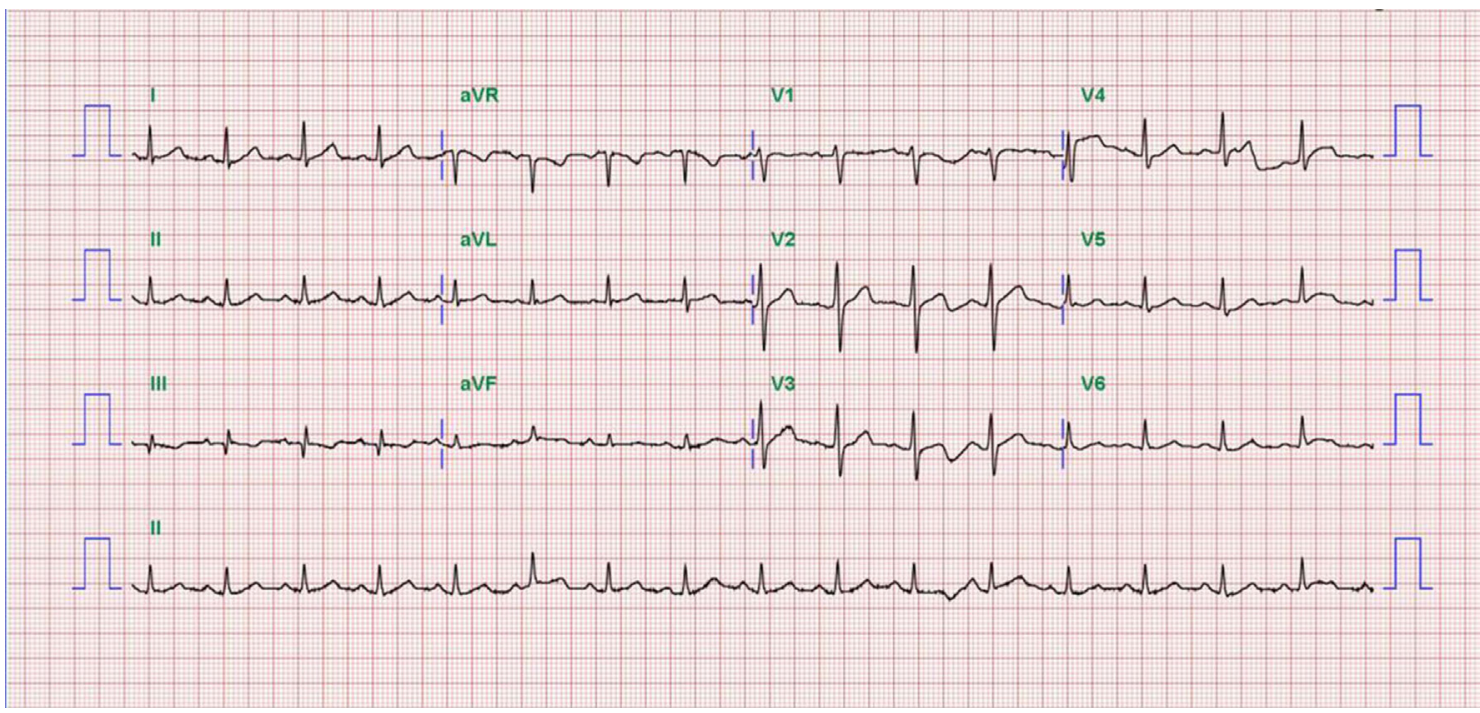

Fig. 5. Electrocardiogram one day after thrombosuction shows ST-segment resolution on II, III, and aVF leads. 
administered, and 60 units $/ \mathrm{kg}$ of unfractionated heparin was injected in the emergency room based on the treatment for STEMI. Coronary angiography (CAG) demonstrated diffuse ectasia in the three major coronary arteries and total occlusion of the distal right coronary artery that was supposed to be just proximal to the bifurcation of the posterior descending artery and posterolateral branch (Fig. 2). Intracoronary infusion of glycoprotein IIb/IIIa inhibitor (abciximab 0.25 $\mathrm{mg} / \mathrm{kg}$ ) and thrombus aspiration were performed. The aspirated material was considered red thrombi (Fig. 3). After aspiration thrombectomy, diffuse huge ectasia-induced sluggish and turbulent blood flow and small attached thrombi without residual stenosis were observed at the previously occluded lesion, and flow grade 3 was obtained (Fig. 4). Therefore, we completed the coronary intervention without further angioplasty and decided to treat the patient conservatively under intensive monitoring, focusing on both ischemic and bleeding events in the coronary care unit (CCU). Dual antiplatelet therapy with aspirin $100 \mathrm{mg}$ qd and ticagrelor $90 \mathrm{mg}$ bid was maintained; atorvastatin $10 \mathrm{mg} \mathrm{qd}$, bisoprolol $2.5 \mathrm{mg} \mathrm{qd}$, and ramipril $2.5 \mathrm{mg}$ qd were added. We decided not to use continuous infusion of abciximab and anticoagulants, as the patient had thrombocytopenia and a prolonged PT. During CCU monitoring, the patient's condition had been stable and the laboratory findings, including platelet count and PT-INR, had not changed significantly. Follow-up electrocardiogram demonstrated ST-segment resolution on II, III, and aVF leads. (Fig. 5) The follow up levels of CK-MB and troponin I were $25.04 \mathrm{ng} / \mathrm{mL}$ and $10,975.5 \mathrm{pg} / \mathrm{mL}$, respectively, and the levels of cardiac markers decreased rapidly during CCU monitoring. Followup CAG 4 days later showed only sluggish and turbulent coronary flow without thrombus. The patient was discharged without any recurrent ischemic events or complications. The patient has continued taking aspirin and ticagrelor for over 6 months without any remarkable changes in laboratory findings.

\section{Discussion}

CAE is a rare condition characterized as the dilatation of a coronary artery to $\geq 1.5$ times the diameter of the adjacent healthy reference segment. CAE differs from coronary ar- tery aneurysm in terms of the shape and extent of coronary artery involvement. The dilation of ectasia is diffuse, involving $>50 \%$ of the major artery, while the dilation of an aneurysm is localized. CAE is an incidental finding in $1-5 \%$ of patients who undergo CAG.[1,2] Although all three major coronary arteries can be affected, only one artery is found to be involved in approximately $75 \%$ of patients. The right coronary artery is most commonly affected, as seen in $60 \%$ cases of whole CAE without a known cause.[7]

CAE is most commonly associated with coronary atherosclerosis; however, $30 \%$ of cases are caused by other conditions, such as congenital connective tissue disorders (Marfan syndrome, Ehlers-Danlos syndrome), infection (syphilis), Kawasaki disease, and other forms of vasculitis. [8] We could not find any other cause of CAE in our patient except for atherosclerosis. Based on etiology, CAE is divided into three types: congenital, acquired atherosclerotic, and isolated or inflammatory. Although the latter type is also acquired, it is not directly related to atherosclerosis. Several conditions, like inflammation, trauma, connective tissue disorders, infections, and the use of certain drugs, lead to this type of ectasia.[9] The atherosclerotic type has been reported to be associated with risk factors such as male sex, hypertension, diabetes mellitus, dyslipidemia, history of stroke, and cigarette smoking, which are similar to those of $\mathrm{CAD}$ in general.[1,3,9]

CAE is often related to endothelial damage and microvascular dysfunction, and dilation of a coronary segment tends to result in stasis, delayed antegrade filling, and segmental back flow. Such conditions cause various manifestations, such as sluggish and turbulent blood flow, coronary vasospasm, spontaneous dissection, or thrombus formation, and these mechanisms can contribute to diverse clinical syndromes.[1,3,7,10] Therefore, the clinical manifestations of CAE can range from asymptomatic or silent ischemia to STEMI as a result of sudden total occlusion by a thrombus. Interestingly, the incidence of myocardial infarction and mortality in atherosclerotic CAE is similar to that of atherosclerotic CAD without ectasia.[1,3]

Meanwhile, a lower incidence of ACS has been reported in patients with chronic liver disease, including liver cirrhosis.[5] Several biochemical changes associated with chronic liver disease might explain the characteristics of less atherosclerotic plaque vulnerability, which is an important factor 
for developing acute coronary events. Coagulation abnormalities, thrombocytopenia, platelet dysfunction, reduced production of total cholesterol and LDL cholesterol, significantly increased activity of nitric oxide, increased plasma estrogen level,[11] and high activity of transforming growth factor beta, a potent inhibitor of the proliferation of cardiac smooth muscle cells,[12] are considered to contribute to the lower incidence of ACS. Those changes also lead to systemic vasodilatation that causes hyperdynamic circulation with greater coronary blood flow and a reduction in systemic vascular resistance in advanced cirrhosis.[5] Traditionally, LC as hypocoagulable status has been thought to be caused by nearly all procoagulant factors being produced less than the anticoagulant factors are produced. The degree of thromobocytopenia and prolongation of PT-INR has been taken as a marker of the severity of coagulopathy.[13]

The development of STEMI in the present case was probably influenced by cardiovascular risk factors related to ectasia, including hypertension, atherosclerosis, and a thrombogenic condition caused by sluggish and turbulent blood flow and spontaneous dissection. Those factors might have preceded the reduced atherosclerotic plaque vulnerability with coagulopathy and the hemodynamic changes following LC.

Treatment guidelines for CAE have not been established due to its rarity and various sizes or types of ectatic coronary arteries. Most of the published treatment options, which are medications, percutaneous intervention, and surgery, have been based on the personal experience of the authors rather than on controlled studies. However, many authors have recommended prophylactic treatment with antiplatelet and anticoagulation therapies due to the high risk of thrombus formation from abnormal flow dynamics.[3,14] Systemic anticoagulation therapy may be strongly indicated for severe coronary artery dilatation (greater than twice the size of the healthy reference diameter), which has been reported to have higher morbidity and mortality rates.[9] Although anticoagulation therapy is important for preventing the recurrence of myocardial infarction in the current case, we decided to use dual-antiplatelet therapy with aspirin and ticagrelor, a potent new $\mathrm{P} 2 \mathrm{Y}_{12}$ inhibitor, considering his mean INR of about 2.0.[15]

Treatment varies depending on the type of CAE, except for antiplatelet and anticoagulation therapies. In our case, LC was another factor to consider when developing the treatment plan. Because patients with atherosclerotic CAE have similar risk factors and mortality rates to patients with atherosclerotic CAD without ectasia, the initial treatment for CAE should be considered to be similar to the treatment for atherosclerotic CAD, as per current guidelines. This includes aggressive control of risk factors such as hypertension, diabetes mellitus, dyslipidemia, and smoking.[3] Medical treatment, including statins, beta blockers, and reninangiotension system inhibitors, are helpful for treating CAE. Statins have been shown to be safe in patients with liver disease, with complication rates similar to those in patients without liver disease.[16] With respect to beta blockers, cardio-selective beta blockers can be used for cardiac comorbidities, such as CAD and congestive heart failure, in early cirrhosis.[17] Limited data suggest that nitroglycerin, even when given sublingually, should be used with caution due to the risk of accelerating ischemia by sluggish coronary blood flow resulting from stasis in CAE patients without stenosis. [14] Other types of congenital and isolated or inflammatory CAE require consultation with specialists for surgical treatment or immunosuppressive and anti-inflammatory drug therapy depending on the underlying etiology.[8]

Surgical and percutaneous approaches for CAE have been reported. The ideal surgical approach is unclear; however, previous data suggested that coronary artery bypass grafting might improve survival rate compared to that for medical therapy.[18] In recent years, the use of the percutaneous approach has increased as technology has developed and improved. Although balloon angioplasty and stent implantation or attempting thrombus aspiration carries the risk of distal thromboembolization, some reports have demonstrated favorable outcomes obtained by successful thrombus removal or using balloon angioplasty with or without stenting.[19] In addition, intracoronary infusion of glycoprotein IIb/IIIa inhibitors is safe and effective for ACS patients who have a large intracoronary thrombus.[20] Therefore, percutaneous revascularization or surgical approaches should be considered depending on the structure or shape of the coronary artery, pathophysiology, and the patient's clinical condition.

We describe a case of STEMI as a result of CAE-related intracoronary thrombus in a patient with LC accompanied by thrombocytopenia and prolonged PT. Patients with CAE can present with various clinical manifestations. Intracoronary thrombus formation within the ectatic segment could 
occur even in patients with abnormal coagulation profiles. Although the guidelines for treatment of CAE have not been established yet, a treatment strategy should be determined under considering the characteristics of the ectatic coronary arteries and the conditions of the patient.

\section{ORCID}

\section{Ji Woong Roh http://orcid.org/0000-0003-0895-5146 \\ Sungmin Lim http://orcid.org/0000-0003-4833-4440}

\section{References}

1) Swaye PS, Fisher LD, Litwin P, Vignola PA, Judkins MP, Kemp HG, et al: Aneurysmal coronary artery disease. Circulation 1983; 67: 134-8.

2) Manginas A, Cokkinos DV: Coronary artery ectasias: imaging, functional assessment and clinical implications. Eur Heart J 2006; 27: 1026-31.

3) Demopoulos VP, Olympios CD, Fakiolas CN, Pissimissis EG, Economides NM, Adamopoulou E, et al: The natural history of aneurysmal coronary artery disease. Heart 1997; 78: 136-41.

4) Yetkin E, Waltenberger J: Novel insights into an old controversy: is coronary artery ectasia a variant of coronary atherosclerosis? Clin Res Cardiol 2007; 96: 331-9.

5) Otsubo R, Higuchi Mde L, Gutierrez PS, Benvenuti LA, Massarollo PC, Costa AL, et al: Influence of chronic liver disease on coronary atherosclerosis vulnerability features. Int J Cardiol 2006; 109: 387-91.

6) Movahed MR, John J, Hashemzadeh M, Jamal MM, Hashemzadeh M: Trends in the age adjusted mortality from acute ST segment elevation myocardial infarction in the United States (1988-2004) based on race, gender, infarct location and comorbidities. Am J Cardiol 2009; 104: 1030-4.

7) al-Harthi SS, Nouh MS, Arafa M, al-Nozha M: Aneurysmal dilatation of the coronary arteries: diagnostic patterns and clinical significance. Int J Cardiol 1991; 30: 191-4.

8) Printz BF, Sleeper LA, Newburger JW, Minich LL, Bradley T, Cohen MS, et al: Noncoronary cardiac ab- normalities are associated with coronary artery dilation and with laboratory inflammatory markers in acute Kawasaki disease. J Am Coll Cardiol 2011; 57: 86-92.

9) Dahhan A: Coronary artery ectasia in atherosclerotic coronary artery disease, inflammatory disorders, and sickle cell disease. Cardiovasc Ther 2015; 33: 79-88.

10) Pinar Bermúdez E, López Palop R, Lozano MartínezLuengas I, Cortés Sánchez R, Carrillo Sáez P, Rodríguez Carreras R, et al: Coronary ectasia: prevalence, and clinical and angiographic characteristics. Rev Esp Cardiol 2003; 56: 473-9.

11) Mendelsohn ME, Karas RH: The protective effects of estrogen on the cardiovascular system. N Engl J Med 1999; 340: 1801-11.

12) Castilla A, Prieto J, Fausto N: Transforming growth factors beta 1 and alpha in chronic liver disease. Effects of interferon alfa therapy. N Engl J Med 1991; 324: 933-40.

13) Arjal R, Trotter JF: International normalized ratio of prothrombin time in the model for end-stage liver disease score: an unreliable measure. Clin Liver Dis 2009; 13: 67-71.

14) Krüger D, Stierle U, Herrmann G, Simon R, Sheikhzadeh A: Exercise-induced myocardial ischemia in isolated coronary artery ectasias and aneurysms ("dilated coronopathy"). J Am Coll Cardiol 1999; 34: 1461-70.

15) Authors/Task Force Members, Roffi M, Patrono C, Collet JP, Mueller C, Valgimigli M, et al: 2015 ESC Guidelines for the management of acute coronary syndromes in patients presenting without persistent STsegment elevation: Task Force for the Management of Acute Coronary Syndromes in Patients Presenting without Persistent ST-Segment Elevation of the European Society of Cardiology (ESC). Eur Heart J 2015. [Epub ahead of print].

16) Tsochatzis EA, Bosch J, Burroughs AK: New therapeutic paradigm for patients with cirrhosis. Hepatology 2012; 56: 1983-92.

17) Ge PS, Runyon BA: The changing role of beta-blocker therapy in patients with cirrhosis. J Hepatol 2014; 60: 643-53.

18) Vijayanagar R, Shafii E, DeSantis M, Waters RS, Desai A: Surgical treatment of coronary aneurysms with and without rupture. J Thorac Cardiovasc Surg 1994; 107: 
1532-5.

19) Oh SK, Rha SW, Kook H, Kim DH, Ho SY, Kim SH, et al: Two different successful angioplasty methods in patients with stenotic coronary artery ectasia. Chonnam Med J 2012; 48: 185-9.

20) Echavarría-Pinto M, Lopes R, Gorgadze T, Gonzalo
N, Hernández R, Jiménez-Quevedo P, et al: Safety and efficacy of intense antithrombotic treatment and percutaneous coronary intervention deferral in patients with large intracoronary thrombus. Am J Cardiol 2013; 111: 1745-50. 